\title{
Heat Shock Protein 72 Antagonizes STAT3 Signaling to Inhibit Fibroblast Accumulation in Renal Fibrogenesis
}

Yi Zhou, ${ }^{* \dagger \ddagger}$ Shirong Cao, ${ }^{* \dagger \ddagger}$ Huiyan Li, ${ }^{* \dagger \ddagger}$ Xuan Peng, ${ }^{* \dagger \ddagger}$ Yating Wang, ${ }^{* \dagger \ddagger}$ Jinjin Fan, ${ }^{* \dagger \ddagger}$ Yihan Wang, ${ }^{\S}$ Shougang Zhuang, Xueqing $\mathrm{Yu}^{*}{ }^{* \dagger}$ and Haiping Mao ${ }^{* \dagger \ddagger}$

From the Department of Nephrology, ${ }^{*}$ The First Affiliated Hospital, Sun Yat-Sen University, Guangzhou, China; the Key Laboratory of Nephrology, ${ }^{\dagger}$ Ministry of Health of China, Guangzhou, China; Guangdong Provincial Key Laboratory of Nephrology, ${ }^{\ddagger}$ Guangzhou, China; the Laboratory for Kidney Pathology, Inc., ${ }^{\S}$ Nashville, Tennessee; and the Department of Medicine," Alpert Medical School and Rhode Island Hospital of Brown University, Providence, Rhode Island

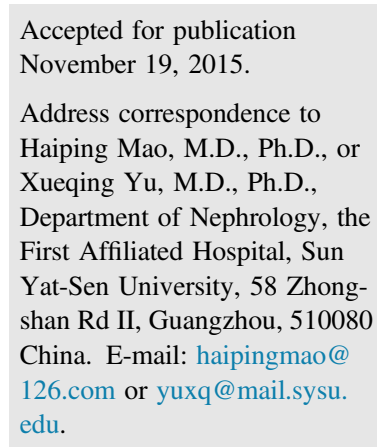

\begin{abstract}
Heat shock protein 72 (HSP72) has been shown to attenuate unilateral ureteral obstruction-induced kidney fibrosis. It remains unknown whether HSP72 has direct effects on fibroblast proliferation in the renal fibrotic evolution. Herein, we first confirmed that increased HSP72 expression occurred in fibrotic human kidneys. Using three different animal models of kidney fibrosis, pharmacological downregulation or genetic deletion of endogenous HSP72 expression exacerbated STAT3 phosphorylation, fibroblast proliferation, and tubulointerstitial fibrosis. In contrast, treatment with geranylgeranyl acetone, a specific inducer of HSP72, reduced phosphorylated STAT3 and protected animals from kidney fibrosis. In cultured renal interstitial fibroblasts, overexpression of HSP72 blocked transforming growth factor (TGF)- $\beta 1$-induced cell activation and proliferation, as evidenced by inhibiting expression of $\alpha$-smooth muscle actin, fibronectin, and collagen I/III, as well as by reducing cell numbers and DNA synthesis. Mechanical studies showed that overexpressed HSP72 attenuated TGF- $\beta$-induced phosphorylation and nuclear translocation of STAT3 and its downstream protein expression. However, siRNA knockdown of HSP72 increased TGF- $\beta$-induced STAT3 activity and fibroblast proliferation. Ectopic expression of a constitutively active STAT3 conferred resistance to HSP72 inhibition of fibroblast proliferation. Thus, HSP72 blocks fibroblast activation and proliferation in renal fibrosis via targeting the STAT3 pathway and may serve as a novel therapeutic agent for chronic kidney disease regardless of the etiology. (Am J Pathol 2016, 186: 816-828; http://dx.doi.org/10.1016/j.ajpath.2015.11.016)
\end{abstract}

Tubulointerstitial fibrosis (TIF) is the histological hallmark of chronic progressive renal disease, characterized by the accumulation of extracellular matrix (ECM) and loss of tubular architecture. The cellular and molecular mechanisms of TIF are still not well clarified. In kidneys with TIF, the cortical interstitium is massively expanded, and the fibroblast acquires a myofibroblast phenotype with expression of proteins, such as $\alpha$-smooth muscle actin ( $\alpha$-SMA), and increased capacity for production of collagen matrix. ${ }^{1}$ Furthermore, activation of fibroblast correlates with development of kidney fibrosis. Although the origin of interstitial activated, matrix-producing fibroblast remains controversial and in debate, many studies conducted in different organs suggest that activation of local resident fibroblast appears to be the principal, if not the only, source of fibroblast recruitment. ${ }^{2,3}$ Thus, therapeutic manipulation of fibroblast proliferation may provide a novel strategy to diminish renal interstitial ECM deposition and the evolution of renal fibrosis.

Multiple signaling molecules have been found to mediate cell proliferation, including STAT3, phosphatidylinositol

Supported by the National Natural Science Foundation of China grants 81070553 (H.M.), 81270783 (H.M.), and 81100491 (Y.Z.); the Natural Science Foundation of Guangdong province grant 10251008901000003 (H.M.); and the National Basic Research Program of China grants 2011CB504005 and 2012CB517700-2012CB517706 (X.Y.).

Disclosures: None declared. 
3-kinase (PI3K)/Akt, and p38 mitogen-activated protein kinase (MAPK). ${ }^{4-9}$ STAT3, a member of the STAT family, is most intimately linked to oncogenic processes, such as proliferation, migration, and differentiation. ${ }^{10}$ STAT3 is activated by phosphorylation of tyrosine residue (Tyr705), which is mediated by growth factor receptor tyrosine kinases, Janus kinases, or the Src family kinases. On activation, STAT3 forms dimer, translocates into the nucleus, and binds to specific DNA response elements, where it promotes the transcription of target genes involved in cell cycle progression, proliferation, and survival. ${ }^{11}$ It has been shown that STAT3 is transiently or persistently activated in human diseases, including tumors and some chronic disease associated with fibrosis. ${ }^{4,5,12-17}$ In addition, patients with constitutive STAT3 activity experienced a particularly poor outcome. ${ }^{15}$ Inhibition of STAT3 phosphorylation has been suggested to attenuate interstitial fibroblast activation and prevent renal fibrogenesis in unilateral ureteral obstruction (UUO) mice. ${ }^{18}$ These findings suggest that STAT3 signaling may play an important role in renal fibroblast proliferation.

As an inducible molecule chaperone, heat shock protein 72 (HSP72) confers cytoprotection against many acute stresses at multiple checkpoints in the cell death pathway. ${ }^{19-23}$ For instance, oral administration of geranylgeranyl acetone (GGA), an antiulcer drug, specifically induces HSP72 expression in the kidney and contributes to the suppression of tubular apoptosis and ischemic acute renal failure. ${ }^{24,25}$ Furthermore, our previous studies have revealed that up-regulation of HSP72 by GGA ameliorates renal fibrosis in a rat UUO model, which counteracts apoptosis and epithelial-mesenchymal transition by interfering with the transforming growth factor (TGF)- $\beta /$ Smads signaling pathway. ${ }^{26,27}$ However, the direct effects of HSP72 on fibroblast proliferation during TIF development have not yet been clarified.

In this study, we examined the expression of HSP72 in human kidney disease with TIF, and performed pharmacological, genetic, in vivo, and in vitro experiments to determine the role of HSP72 in interstitial fibroblast activation and proliferation during chronic TIF.

\section{Materials and Methods}

\section{Reagents}

Antibodies against STAT3, phosphorylated (p)-STAT3 ${ }^{\text {tyr705, }}$ p38, Akt, p-p38, and p-Akt were from Cell Signaling Technology (Beverly, MA). Antibodies to cyclin D1, p21, proliferating cell nuclear antigen, and $\beta$-actin were from UpState Biotechnology (Waltham, MA). Antibodies to $\alpha$-SMA, fibronectin, and collagen I/III were from Santa Cruz Biotechnology (Dallas, TX). Anti-HSP72 was from Stressgen Biotechnologies (Victoria, BC, Canada). Anti-fibrillarin was from Abcam (Cambridge, MA). Wortmannin and SB203580 were from EMD Chemicals (Gibbstown, NJ). Recombinant human TGF- $\beta 1$ was from R\&D Systems (Minneapolis, MN).
Alexa Fluor 546-conjugated anti-rabbit IgG, Alexa Fluor 633-conjugated anti-mouse $\operatorname{IgG}$, and Alexa Fluor 488-conjugated anti-rabbit IgG were from Thermo Fisher Scientific (Rockford, IL). NE-PER nuclear and cytoplasmic extraction reagents were from Pierce Biotechnology (Rockford, IL). Quercetin was purchased from Sigma-Aldrich (St. Louis, MO). GGA was from Eisai China Inc. (Shanghai, China). All other reagents were from Sigma-Aldrich.

\section{Human Kidney Tissues}

Tissue specimens were obtained from archived kidney biopsy specimens and nephrectomies at the Department of Nephrology, the First Affiliated Hospital of Sun Yat-Sen University (Guangzhou, China). Fibrosis was assessed by Masson's trichrome staining. Human kidney specimens were collected from biopsy-proved chronic kidney disease (CKD) with substantial TIF $(n=10)$. Normal control renal tissues were from disused donor kidneys, which were determined unsuitable for transplantation $(n=4)$. Clinical data were documented at the time of renal biopsy and are described in Supplemental Table S1. The estimated glomerular filtration rate was calculated from serum creatinine by the modification of diet in renal disease equation. CKD was defined by Kidney Disease Outcomes Quality Initiative guidelines. ${ }^{28}$ The study was approved by the Ethics Committee of the First Affiliated Hospital, Sun Yat-Sen University, and written informed consent was obtained from all subjects before kidney biopsy or nephrectomy.

\section{Induction of Kidney Fibrosis in Animals}

Male Sprague-Dawley rats (200 to $250 \mathrm{~g}$ ) were obtained from the Guangdong Medical Experimental Animal Center (Guangzhou, China). To examine the role of HSP72 in fibroblast proliferation, rats were given GGA or GGA plus quercetin treatment to pharmacologically regulate HSP72 expression and were subjected to UUO, as described previously. ${ }^{29}$ Briefly, to optimally induce HSP72 expression, $400 \mathrm{mg} / \mathrm{kg} \mathrm{GGA}$ or vehicle (dimethyl sulfoxide) was orally administered to rats 1 day before UUO and daily for 7 days. For GGA/quercetin combination treatment, rats were injected i.p. with $100 \mathrm{mg} / \mathrm{kg}$ quercetin or vehicle (saline) 1 hour before GGA administration. Furthermore, to determine the specific protective effects of HSP72 in kidney injury, additional studies, including UUO, unilateral ischemia reperfusion injury, and acute aristolochic acid nephropathy, were conducted on knockout mice deficient in HSP72 expression. HSP72 wild-type mice on a C57BL/6J background from Mutant Mouse Regional Resource Centers at The Jackson Laboratory (Bar Harbor, ME) were intercrossed to produce $H S P 72^{-/-}$and wild-type $\left(H S P 72^{+/+}\right)$offspring. The genotype of the mice was identified by genomic PCR analysis using specific primers. Experiments were conducted using male mice aged 8 to 10 weeks, weighting 20 to $25 \mathrm{~g}$. 
The UUO model was performed by ligation of the left ureter, and sham-operated on animals were used as controls, as previously reported. ${ }^{26}$ Groups of mice or rats $(n=6)$ were sacrificed at day 7 after UUO. Kidney ischemia injury was induced by occluding the left renal pedicles with a vascular clamp for 30 minutes at $37^{\circ} \mathrm{C}$. The sham operations were performed in an identical manner, except for clamping of the renal pedicles. ${ }^{30}$ The aristolochic acid nephropathy model was induced by one-time i.p. injection of $5 \mathrm{mg} / \mathrm{kg}$ aristolochic acid (Sigma) in phosphate-buffered saline. ${ }^{31}$ The same volume of phosphate-buffered saline was given in the control mice. Groups of mice $(n=6)$ were sacrificed at day 42 after ischemia or injection of aristolochic acid. Kidney tissues were harvested for routine pathological analysis. Collagen accumulation was determined by Sirius redor Masson's trichrome staining and quantified by image analysis. Histological evaluations were assessed in a blinded manner (J.F.). All animal studies were approved by the Animal Care and Use Committee of the Sun Yat-Sen University.

\section{Immunofluorescence Staining}

Immunofluorescence staining for paraffin-embedded kidney sections and cultured cells was performed as previously described ${ }^{26,27}$ For triple staining, tissue sections were blocked with $10 \%$ donkey serum in phosphate-buffered saline for 1 hour at room temperature, incubated with rabbit anti-human p-STAT3 ${ }^{\text {tyr705 }}$ antibody and mouse anti-human $\alpha$-SMA antibody at $4^{\circ} \mathrm{C}$ overnight, and followed by their corresponding secondary antibodies, including Alexa Fluor 546-conjugated anti-rabbit IgG and Alexa Fluor 633-conjugated anti-mouse IgG. The sections were then incubated with rabbit antihuman HSP72 antibody at $4^{\circ} \mathrm{C}$ overnight after being reblocked with $10 \%$ serum for 1 hour, followed by Alexa Fluor 488-conjugated anti-rabbit IgG. The kidney sections were counterstained with DAPI and imaged with a confocal laser-scanning microscope (Zeiss LSM 510 META; Carl Zeiss, Oberkochen, Germany).

Renal interstitial myofibroblasts were identified by positive staining with antibody against $\alpha$-SMA. For the quantification of p-STAT3 or $\alpha$-SMA positivity, 20 random and consecutive images were taken from the renal cortex at $\times 400$ magnification. The percentage of positive staining was extracted for intensity and analyzed with ImageJ software version $1.43 \mathrm{j}(\mathrm{NIH}$, Bethesda, MD). A mean area was calculated from the 20 images of an individual subject.

\section{Plasmid Transfection and RNA Interference}

HSP72 expression vector and HSP72 siRNA construct were described previously. ${ }^{30}$ The STAT3-C construct was made by substituting cysteine residues for A662 and N664 of murine STAT3, which allowed for the sulfhydryl bond to form between STAT3 monomers and rendered the STAT3-C capable of dimerizing without a phosphate on Y705. ${ }^{32}$ The human STAT3 siRNA and scrambled siRNA were purchased from Shanghai
GenePharma Co, Ltd (Shanghai, China). The sequences of each siRNA were as follows: scrambled siRNA (sense, $5^{\prime}$-CCUACGCCACCAAUUUCGUDTDT-3'; antisense, 5'-ACGAAAUUGGUGGCGUAGGDTDT- $3^{\prime}$ ) and Stat 3 siRNA (sense, 5'-CUAUCUAAGCCCUAGGUUUDTDT-3'; antisense, $5^{\prime}$-AAACCUAGGGCUUAGAUAGDTDT- ${ }^{\prime}$ ). Cells were transfected with the indicated concentrations of either STAT3-specific or scrambled siRNA with Lipofectamine RNAiMAX in Opti-mem (Invitrogen, Grand Island, NY), according to the manufacturer's instructions. Overexpression or knockdown efficacy was assessed by Western blot analysis.

\section{Cell Culture and Treatments}

NRK-49F cells were obtained from ATCC (Manassas, VA). Cells were cultured in Dulbecco's modified Eagle's medium mixed 1:1 with F12 medium (Invitrogen) supplemented with $10 \%$ fetal bovine serum. Recombinant human TGF- $\beta 1$ was added to the culture at different concentration for various time periods, as indicated. In some experiments, cells were pretreated with $10 \mu \mathrm{mol} / \mathrm{L}$ various chemical inhibitors or vehicle control (dimethyl sulfoxide) for 30 minutes, followed by incubation with $2 \mathrm{ng} / \mathrm{mL}$ TGF- $\beta 1$ for an additional 24 hours.

\section{Western Blot Analysis}

Proteins were isolated from kidney tissue or cell extracts and homogenized in lysis buffer, as previously reported. ${ }^{27}$ After centrifugation to remove debris, supernatant was subjected to protein assay. Samples were loaded and separated by $10 \%$ SDS polyacrylamide gels, electrotransferred onto a nitrocellulose membrane, blotted with indicated antibodies, and then detected by enhanced chemiluminescence (Amersham Pharmacia Biotech, Piscataway, NJ).

\section{Cell Proliferation and DNA Synthesis Assay}

Total cell numbers were counted by using a hemocytometer. DNA synthesis was determined by measuring bromodeoxyuridine incorporation into DNA via a nonradioactive colorimetric assay using an enzyme-linked immunosorbent assay (Roche Diagnostics West Sussex, UK), according to the manufacturer's protocol. Briefly, $2.5 \times 10^{4}$ cells were seeded in triplicate in 96-well flat-bottomed plates and allowed to adhere for 48 hours. Cells were transiently transfected with HSP72 plasmid or HSP72 siRNA, followed by incubation with $2 \mathrm{ng} / \mathrm{mL}$ of TGF- $\beta 1$ for 24 hours. Results are expressed as the percentage of control from at least three independent experiments.

\section{Native Gel PAGE}

To determine Stat3-dimer formation, cells were lysed in icecold isotonic buffer [ $20 \mathrm{mmol} / \mathrm{L}$ Tris $(\mathrm{pH} 7.0), 150 \mathrm{mmol} / \mathrm{L}$ $\mathrm{NaCl}, 6 \mathrm{mmol} / \mathrm{L} \mathrm{MgCl}_{2}, 0.8 \mathrm{mmol} / \mathrm{L}$ phenylmethylsulfonyl 

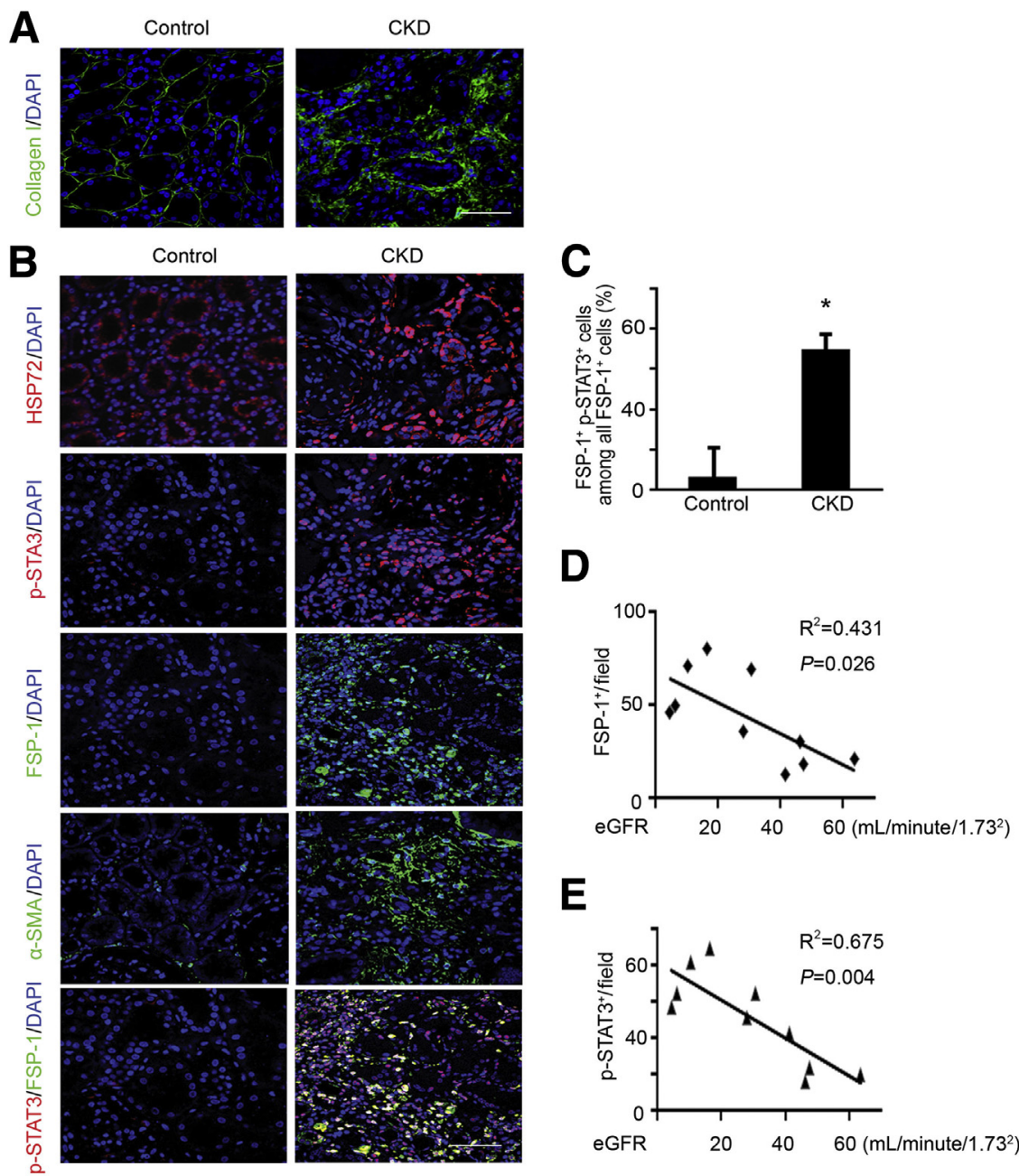

E

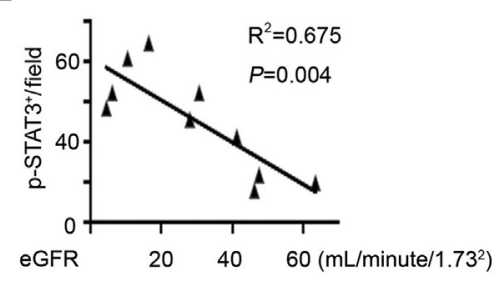

Figure 1 Increased expression of heat shock protein (HSP) 72 and phosphorylated (p)-STAT3 in kidneys of patients with tubulointerstitial fibrosis. A: Representative images of collagen I from a control healthy individual and from a patient with chronic kidney disease (CKD). B: Representative images of immunofluorescence labeling for HSP72 (green), p-STAT3 (red), $\alpha$-smooth muscle actin ( $\alpha$-SMA; purple), and DAPI (blue) on kidney sections. C: The percentage of $\alpha$-SMA ${ }^{+}$p-STAT3 ${ }^{+}$ cells among total $\alpha-\mathrm{SMA}^{+}$fibroblasts in different groups. D and E: The number of cells with positive $\alpha$-SMA and p-STAT3 staining negatively correlates with the estimated glomerular filtration rate (eGFR), respectively. Data represent means \pm SEM (C). ${ }^{*} P<0.05$ versus healthy control. Scale bar $=$ $50 \mu \mathrm{m}$ (A and B). FSP-1, fibroblast-specific protein-1.

fluoride, and $20 \%$ glycerol]. Cell extracts were separated onto 6\% SDS-free PAGE gels, transferred to polyvinylidene difluoride membranes, and immunoblotted with either STAT3 or HSP72 antibody, as described by Kaissling and Le Hir. ${ }^{33}$

\section{Electrophoretic Mobility Shift Assay}

Nuclear protein extracts were prepared from NRK-49F cells, as described previously. ${ }^{30}$ Nuclear extracts were incubated for 20 minutes at room temperature with the biotin-labeled, doublestranded STAT3 consensus-binding motif: high-affinity c-sis inducible element consensus sequence (sense, 5'-AGCTTCATTTCCCGTAAATCCCTA- $3^{\prime}$ ) derived from the fos gene promoter. The specificity of the DNA-protein interaction was established by competition experiments using unlabeled oligonucleotide as the competitor. Binding reactions and electrophoresis were performed using the LightShift Chemiluminescent electrophoretic mobility shift assay kit (Pierce Biotechnology, Inc. Rockford, IL), according to the manufacturer's protocol. Protein-DNA complexes were resolved onto $6 \%$ nondenaturing polyacrylamide gels and transferred to nylon membranes. The DNA was crosslinked, and the biotin-labeled DNA was detected by chemiluminescence.

\section{Transient Transfection and Dual-Luciferase Assay}

STAT3 transcriptional activity was examined by transient transfection assays of the pSTAT3-Luc reporter plasmid (STAT3-Luc). The pTA-Luc plasmid without carrying STAT3 responsible DNA elements was used as a control. Cells were transfected with pSTAT3-TA-Luc and pRL-TK, a Renilla luciferase control reporter plasmid (Promega, Madison, WI) using TransFectin (Bio-Rad Laboratories, Philadelphia, PA). After 5 hours of transfection, cells were incubated with complete medium for 24 hours and then introduced with wt-HSP72 or siRNA. Luciferase activity was evaluated by the Dual Luciferase Reporter Assay kit (Promega). The activity was normalized as to transfection efficiency using the Renilla luciferase activity of pRL-TK. Experiments were performed in triplicate and at least five independent times.

\section{Statistical Analysis}

All results are expressed as means \pm SEM. Groups were compared with one-way analysis of variance, followed by the Student-Newman-Keuls test. The Spearman correlation coefficient test was used to evaluate associations between two quantitative variables. $P<0.05$ was considered significant. 
A
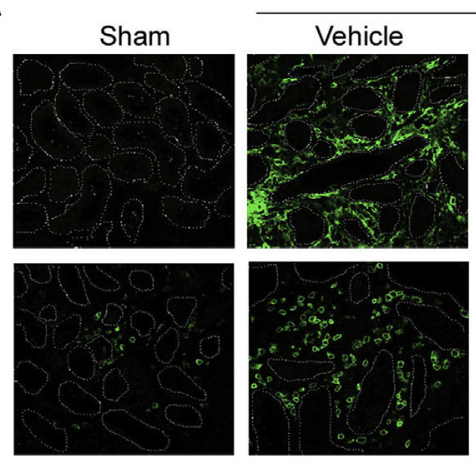

B
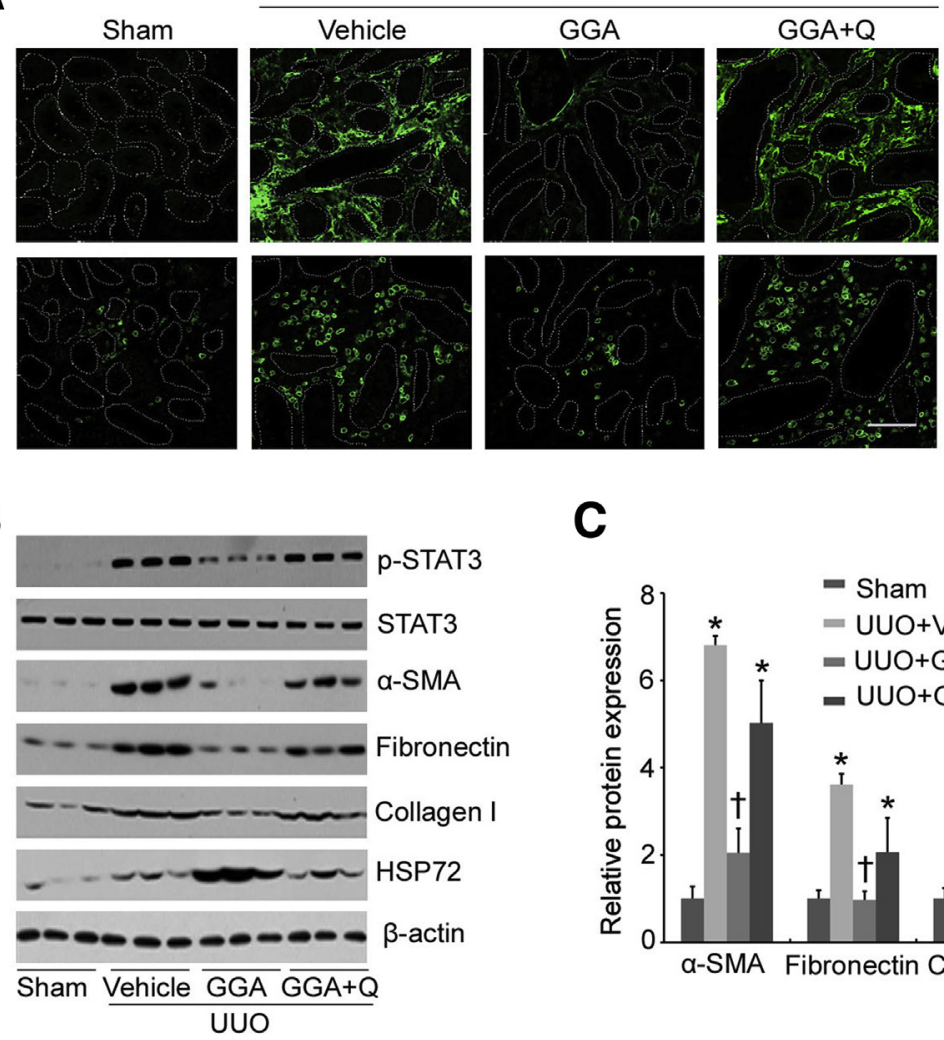

C

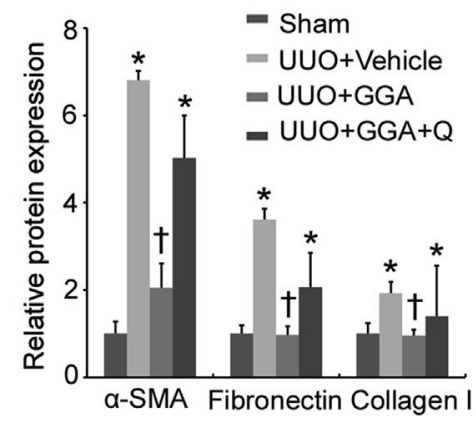

UUO
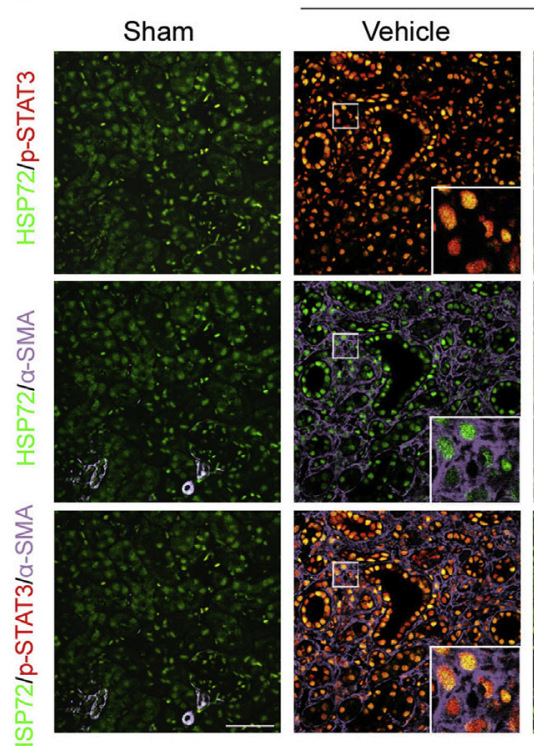

a-SMA

Fibronectin

Figure 2 Geranylgeranyl acetone (GGA) ameliorates unilateral ureteral obstruction (UUO)-induced fibroblast activation. A: Representative immunofluorescence staining of $\alpha$-smooth muscle actin $(\alpha-S M A)$ and fibronectin on kidney sections from sham - or UU0-operated on rats with vehicle, GGA, or GGA plus quercetin ( $Q$ ) treatment. B: Expression of heat shock protein (HSP) 72 in kidneys is up- and down-regulated by GGA and $Q$ administration, respectively, as described in Materials and Methods. Immunoblot analyses for the indicated proteins. C: Relative protein amounts of $\alpha$-SMA, fibronectin, and collagen I in kidney tissues of UUO-induced rats are reported after normalization with $\beta$-actin. D: Representative immunofluorescence staining of HSP72 (green), p-STAT3 (red), $\alpha$-SMA (purple), and DAPI (blue) on kidney sections from different groups. Boxed areas in $\mathbf{D}$ are enlarged and represented in the insets. The images are representative of multiple experiments. Values are means \pm SEM (C). $n=6$ (C and $\mathbf{D}) .{ }^{*} P<0.05$ versus sham groups; ${ }^{\dagger} P<0.05$ versus vehicle-treated or GGA plus $Q$-treated groups. Scale bar $=50 \mu \mathrm{m}(\mathbf{A}$ and $\mathbf{D})$.

\section{Results}

Expression of HSP72 and p-STAT3 Is Elevated in Human Kidney Fibrosis

Immunofluorescence analysis displayed that collagen I deposition was dramatically increased in human fibrotic kidneys compared with nonfibrotic control kidneys
(Figure 1A). Furthermore, in the nonfibrotic kidneys, the enrichment of HSP72 was observed in the cytosol of tubular cells, whereas p-STAT3 was not detectable and $\alpha$ SMA was barely expressed. In contrast, the expression of HSP72, p-STAT3, and $\alpha$-SMA was increased in fibrotic kidneys. Enhanced HSP72 expression was predominantly present in the nuclei of both tubular cells and $\alpha$-SMApositive myofibroblasts, where it colocalized with STAT3 


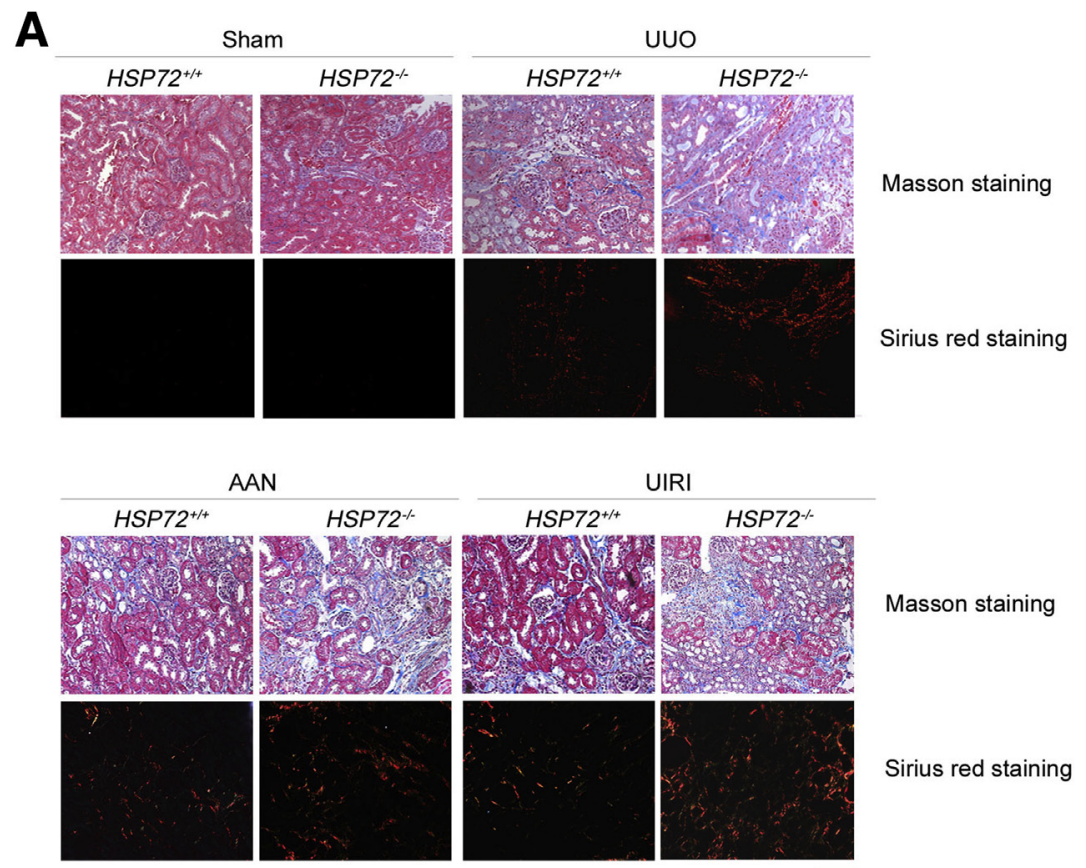

B
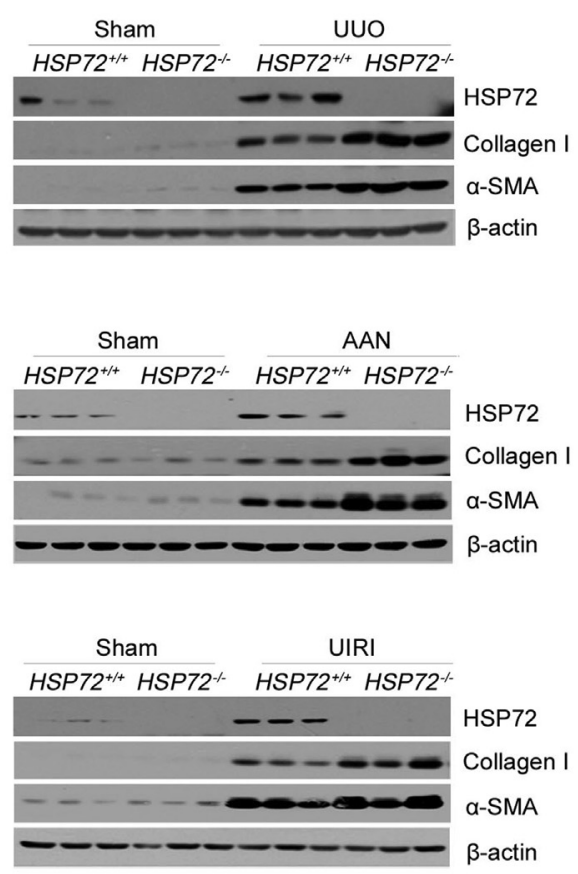
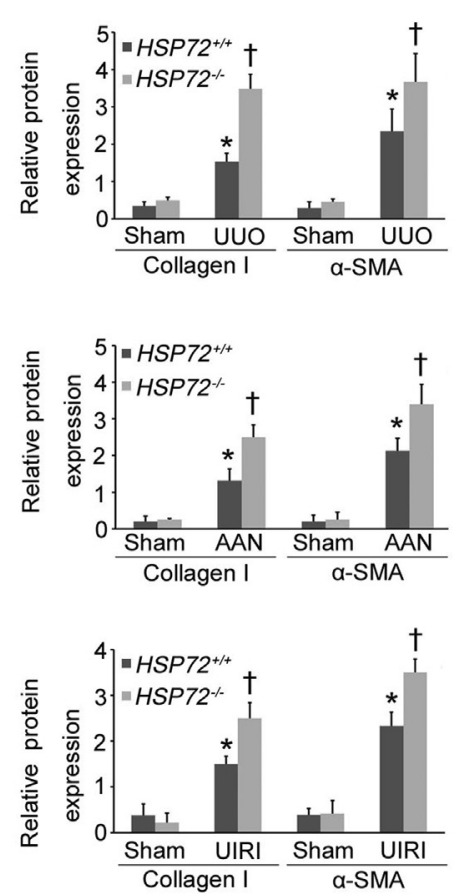

Figure 3 Deficiency of heat shock protein (HSP) 72 enhances renal fibrosis in three fibrotic animal models. A: Representative images of Masson's trichrome and Sirius red staining on sham and fibrotic kidneys of unilateral ureteral obstruction (UUO), acute aristolochic acid nephropathy (AAN), and unilateral ischemia reperfusion injury (UIRI). B: Immunoblot analyses for the indicated proteins in the entire kidney of $H S P 72^{-/-}$and $\mathrm{HSP}^{+/+}$mice from three mice models. Relative protein amounts of HSP72, collagen I, and $\alpha$-smooth muscle actin ( $\alpha$-SMA) in kidney tissues are reported after normalization with $\beta$-actin. Values are means $\pm \operatorname{SEM}(B) . n=6$ (B). ${ }^{*} P<0.05$ versus sham groups; ${ }^{\dagger} P<0.05$ versus $\mathrm{HSP}^{+/+}$mice. Scale bar $=50 \mu \mathrm{m}(\mathbf{A})$.
(Figure 1B). The percentage of both $\mathrm{p}-S T A T^{+}$and $\alpha-\mathrm{SMA}^{+}$fibroblasts among all $\alpha$-SMA ${ }^{+}$fibroblasts was significantly higher in fibrotic kidneys than in the nonfibrotic control kidneys $(72.25 \%$ versus $1.33 \%$; $P<0.05)$ (Figure 1C), suggesting that STAT3 phosphorylation is associated with fibroblast accumulation during the renal fibrogenesis. Statistical analysis showed that the expression of $\alpha$-SMA $\left(R^{2}=0.61, P=0.008\right)$ (Figure 1D) and p-STAT3 $\left(R^{2}=0.68, P=0.004\right)$ (Figure $1 \mathrm{E}$ ) negatively correlated with the estimated glomerular filtration rate.
Pharmacological Induction of HSP72 Ameliorates Fibroblast Accumulation and STAT3 Activation in a Rat UUO Model

GGA has been shown to induce expression of HSP72, and quercetin is an inhibitor of HSP72. ${ }^{24,27,29,34}$ To gain insight into the role of HSP72 in fibroblast proliferation and STAT3 phosphorylation in the development of kidney fibrosis, we treated rats with GGA or GGA plus quercetin before UUO, as previously described. ${ }^{26,35}$ By immunofluorescence staining, 
A

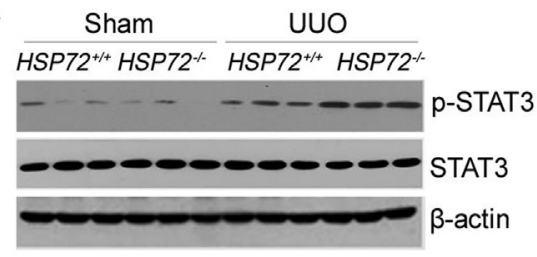
$\frac{\text { Sham }}{\mathrm{HSP}^{+/+} H S P 72^{-\leftarrow}} \frac{\text { AAN }}{H S P 72^{++\alpha} H S P 72^{-}}$ $-\approx=-\sigma=\mathrm{m}$-STAT3 -0000000000 STAT3 $\beta$-actin
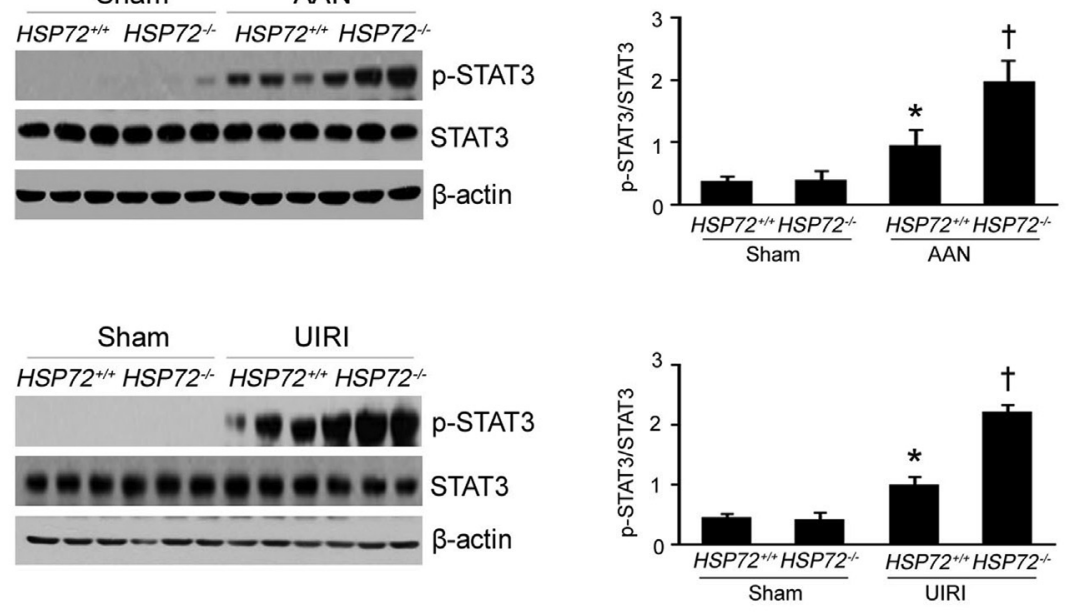

B

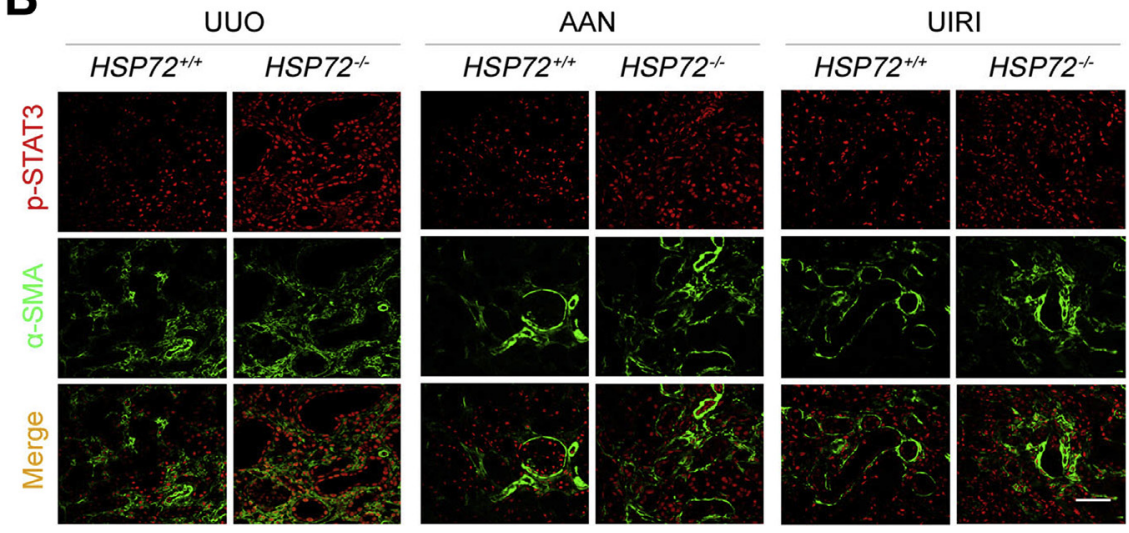

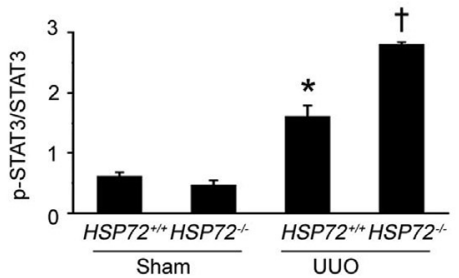

Figure 4 Heat shock protein (HSP) 72 knockout promotes STAT3 activation in fibrotic mice models. A: Immunoblot analyses of phosphorylated (p)-STAT3 and STAT3 in the sham and fibrotic kidneys of mice. $\beta$-Actin was used as an internal control. The histogram shows the relative band intensities normalized to STAT3. B: Representative immunofluorescence images of p-STAT3 (red) and $\alpha$-smooth muscle actin ( $\alpha$-SMA; green) on kidney sections in different groups, as indicated. Data represent means $\pm \operatorname{SEM}(\mathbf{A}) . n=6$ (A and $\mathbf{B}) .{ }^{*} P<0.05$ versus sham; ${ }^{\dagger} P<0.05$ versus $\mathrm{HSP}^{+/+}$mice. Scale bar $=50 \mu \mathrm{m}$ (B). AAN, aristolochic acid nephropathy; UIRI, unilateral ischemia reperfusion injury; UU0, unilateral ureteral obstruction.
GGA-treated rats showed significantly decreased expression of $\alpha$-SMA and fibronectin in the obstructed kidneys at day 7 after UUO, compared with the vehicle control and GGA plus quercetin-administrated rats (Figure 2A). These results were confirmed by lower levels of the markers associated with fibrosis, including $\alpha$-SMA, fibronectin, and collagen $I$ in the GGA-treated rats, as determined by immunoblot analysis. Furthermore, GGA treatment suppressed UUO-induced STAT3 phosphorylation. The GGA inhibitory effects were abrogated by blockade of HSP72 with quercetin (Figure 2, B and $\mathrm{C}$ ). Notably, consistent with our previous observation, oral administration of GGA markedly increased the expression of HSP72, whereas quercetin treatment blocked the upregulation of $\mathrm{HSP} 72$ by GGA. Immunostaining results showed that the accumulation of $\mathrm{p}_{-\mathrm{STAT}^{+}}{ }^{\alpha-\mathrm{SMA}^{+}}$ fibroblast was accompanied by an exaggerated fibrosis in the obstructed kidneys of vehicle-treated rats. Enhanced HSP72 expression by GGA reduced the accumulation of p-STAT3 ${ }^{+}$ $\alpha-\mathrm{SMA}^{+}$fibroblasts, whereas quercetin coadministered with GGA reversed GGA inhibitory effects on proliferation of p-STAT3 $^{+}$fibroblasts (Figure 2D). In addition, the expression patterns of HSP72 and p-STAT3 were similar to those obtained in fibrotic human kidneys and revealed that overexpressed HSP72 alleviated STAT3 phosphorylation not only in the renal tubular cells, but also in the $\alpha-\mathrm{SMA}^{+}$ myofibroblasts.

\section{HSP72 Knockout Exacerbates Renal Fibrosis in Different Fibrotic Animal Models}

To further investigate the role of HSP72 in kidney fibrosis, we used HSP72-null $\left(\mathrm{HSP}^{-/-}\right)$and wild-type littermate 
A
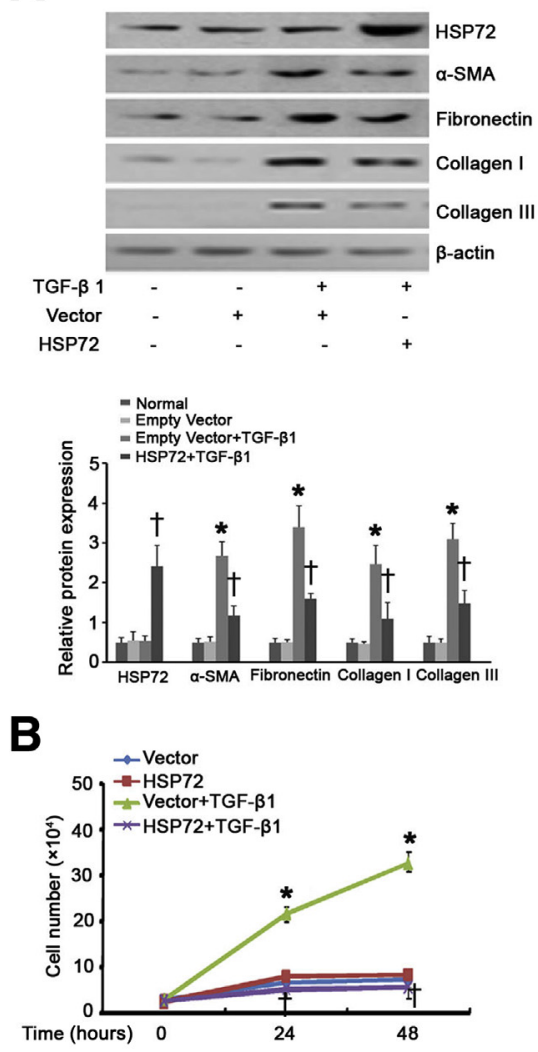

C

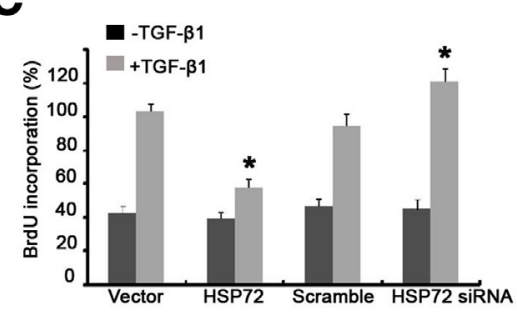

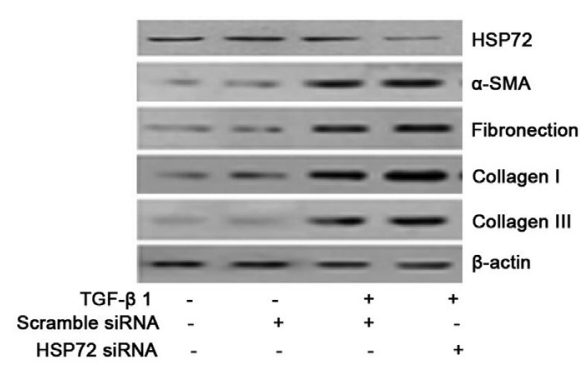
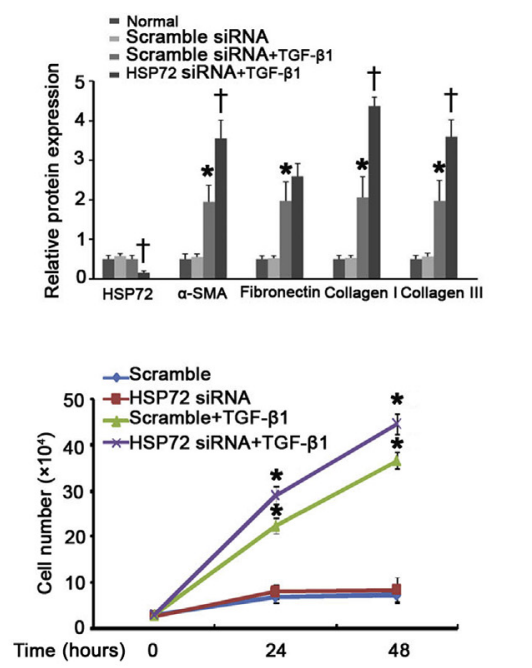

D

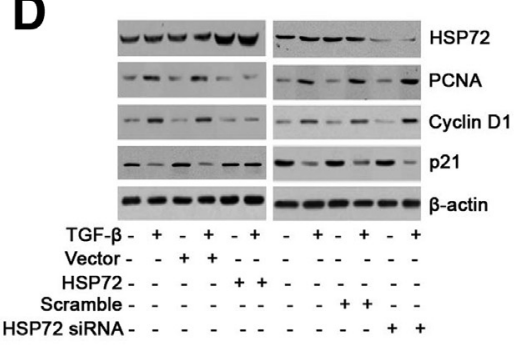

Figure 5 Heat shock protein (HSP) 72 abrogates fibroblast activation and proliferation in vitro. A: NRK-49F cells transfected with wildtype (wt)-HSP72 or HSP72 siRNA were stimulated with $2 \mathrm{ng} / \mathrm{mL}$ of transforming growth factor (TGF)$\beta 1$ for 24 hours. Empty vector or scramble siRNA served as negative control. Expression of the indicated proteins was examined by immunoblot analysis. $\beta$-Actin was used as a loading control. Quantitative determination of the relative abundance of the indicated proteins after normalization with $\beta$-actin among different groups. B: Cells were transfected with empty vector, wt-HSP72, scramble siRNA, or HSP72 siRNA and challenged with $2 \mathrm{ng} / \mathrm{mL}$ TGF- $\beta 1$ for the indicated time period. The total number of cells from each well was counted using a hemocytometer. C: Bromodeoxyuridine (BrdU) incorporation into DNA of cells after various treatments represented as percentage of control with TGF- $\beta 1$ exposure alone. D: Cell lysates after various treatments were subjected to immunoblot analysis using antibodies against the indicated proteins. Data are means $\pm \operatorname{SEM}(\mathbf{A}$ and C). $n=4$ (A); $n=5$ (B); $n=3$ (C). ${ }^{*} P<0.05$ versus empty vector or scramble siRNA control; ${ }^{\dagger} P<0.05$ versus TGF- $\beta 1$-treated cells alone. PCNA, proliferating cell nuclear antigen; $\alpha$-SMA, $\alpha$-smooth muscle actin.
$\left(H S P 72^{+/+}\right)$mice for three different animal models of renal fibrosis: UUO, unilateral ischemia reperfusion injury, and acute aristolochic acid toxic nephropathy. ${ }^{26,31}$ By Masson's trichrome staining and Picrosirius red staining (Figure 3A), there was greater collagen deposition and fibrosis in $H S P 72^{-/-}$mice kidneys than sham-operated on and $H S P 72^{+/+}$control mice in all three models. Consistent with histological findings, immunoblot analysis also confirmed that HSP72 deficiency significantly enhanced protein levels of collagen I and $\alpha$-SMA in the fibrotic kidneys in contrast to $H S P 72^{+/+}$control mice (Figure 3B). Notably, HSP72 protein expression was not detectable in the kidney of homozygous HSP72 knockout mice. Moreover, immunoblot analysis revealed a significant increase in p-STAT3 contents in the kidneys of $H S P 72^{-1-}$ mice compared with sham-operated on mice and $\mathrm{HSP}^{+/+}$control mice (Figure 4A). Examination of kidney sections by immunofluorescence staining showed that the number of cells with positive p-STAT3 and $\alpha$-SMA staining was more in $H S P 72^{-/-}$ mice than those in $\mathrm{HSP}^{+/+}$and sham-operated on mice (data not shown) in all three models (Figure 4B). In addition, histology and protein expression in kidneys from shamoperated on or vehicle-treated mice did not show changes (data not shown). Taken together, HSP72 deficiency aggravated STAT3 phosphorylation and kidney fibrosis, indicating that HSP72 could be an important inhibitor of fibroblast accumulation in the development of kidney fibrosis, likely through blocking of STAT3 phosphorylation.

\section{HSP72 Inhibits Fibroblast Activation and Proliferation in Vitro}

Given the protective role of HSP72 in kidney fibrosis, we next explored whether HSP72 directly blunts fibroblast activation and proliferation in vitro. To this end, cultured fibroblast cells (NRK-49F) were transiently transfected with plasmid vectors encoding HA-epitope tagged-Wt-HSP72- or HSP72-specific 
A
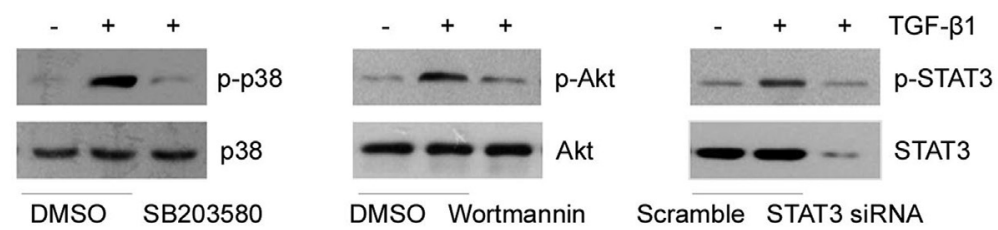

B
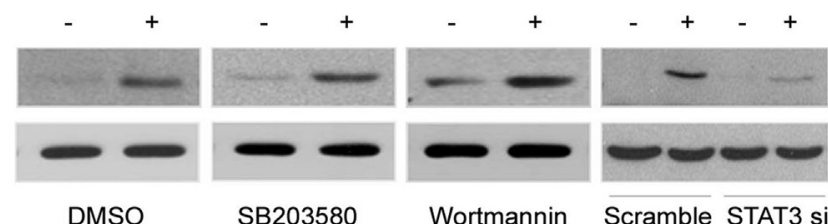

TGF- $\beta 1$

SB203580

Wortmannin

Scramble $\overline{\text { STAT3 siRNA }}$
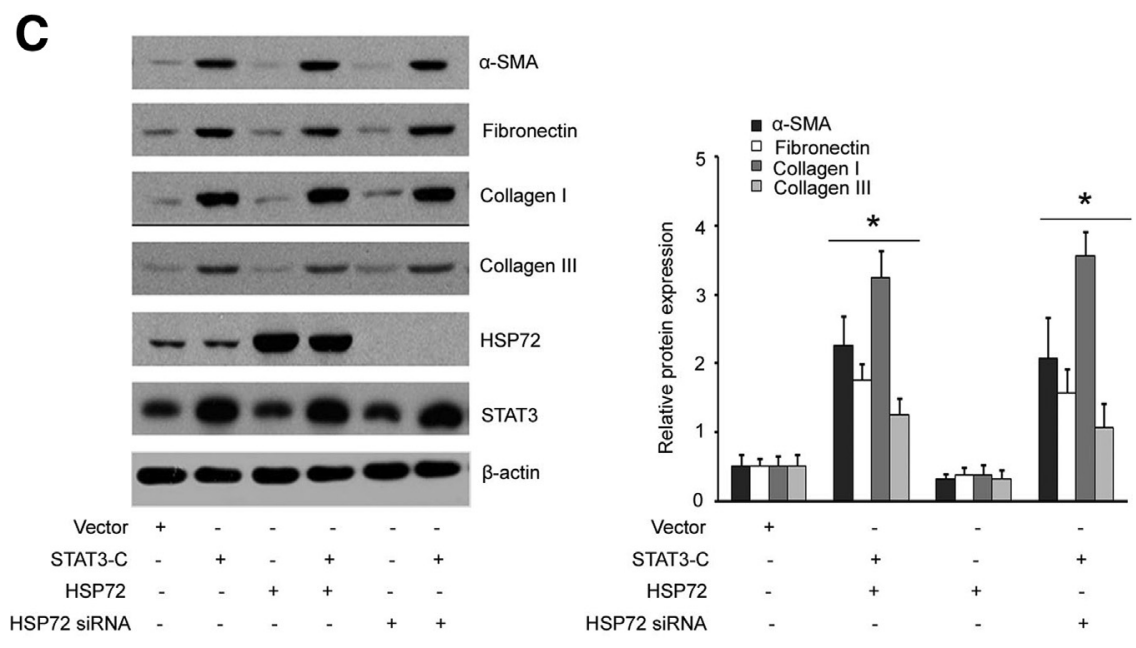

Figure 6 Heat shock protein (HSP) 72mediated inhibition of fibroblast activation depends on STAT3 dephosphorylation. A: Cells pretreated with SB203580 or wortmannin or transfected with STAT3 siRNA were incubated with transforming growth factor (TGF)- $\beta 1$ for 30 minutes. Cell lysates were probed with antibodies against the indicated proteins. B: Cell lysates after treatment with TGF- $\beta 1$ for 30 minutes were subjected to immunoblot analysis using antibodies against the indicated proteins. C: NRK- $49 \mathrm{~F}$ cells were transfected with or without STAT3-C, wild-type (wt)-HSP72, or HSP72 siRNA. Cell lysates were immunoblotted with antibodies against the indicated proteins. Quantitative data show the indicated protein abundances after various treatments, corrected with $\beta$-actin content. ${ }^{*} P<0.05$ versus empty vector control or wt-HSP72 vector treated alone. $n=3(\mathbf{C})$. DMSO, dimethyl sulfoxide; $\alpha$-SMA, $\alpha$-smooth muscle actin.

siRNA, as previously reported. ${ }^{27}$ Compared with empty vector, up-regulation of HSP72 attenuated TGF- $\beta 1$-induced fibroblast activation, as indicated by suppressing the expression of $\alpha$-SMA, fibronectin, and collagen I/III, whereas HSP72 silencing enhanced the ability of TGF- $\beta 1$ in fibroblast activation (Figure 5A). Consistently, elevated expression of HSP72 blocked TGF- $\beta 1$-induced fibroblast proliferation, as indicated by a marked reduction in the cell number (20.3 versus 1.7; $P<0.05$ ) (Figure $5 \mathrm{~B}$ ) and the bromodeoxyuridine incorporation (103.3 versus 58; $P<0.05$ ) (Figure 5C). Inhibition of fibroblast proliferation was accompanied by decreased proliferating cell nuclear antigen and cyclin D1 expression and increased p21 contents (Figure 5D). However, all above responses to TGF- $\beta 1$ stimulation were abolished or substantially reduced by HSP72 siRNA treatment. These data are in agreement with the in vivo findings and support an important functional role for HSP72 in blocking fibroblast activation and proliferation.

\section{Inhibition of STAT3 Phosphorylation Is Critical for} HSP72-Mediated Suppression of Fibroblast Activation and Proliferation

STAT3, PI3K/Akt, and p38 MAPK are major signaling molecules involved in cell proliferation and survival. TGF$\beta 1$ increased the phosphorylation of Akt, p38, and STAT3 in
NRK-49F cells (Figure 6A). Knockdown of STAT3 with specific siRNA inhibited TGF- $\beta 1-$ induced $\alpha$-SMA expression (Figure 6B). However, this response was not attenuated by pretreatment with wortmannin (PI3K/Akt inhibitor) or SB203580 (p38 inhibitor) (Figure 6B), suggesting that activation of STAT3, but not the PI3K/Akt or p38 MAPK signal pathway, contributes to TGF- $\beta 1$-induced fibroblast activation.

To determine whether STAT3 phosphorylation is essential for HSP72 to suppress fibroblast activation, we introduced a constitutively active form of STAT3 (STAT3-C) into NRK-49F cells. As anticipated, expression of STAT3-C induced fibroblast activation, as demonstrated by fibrotic protein induction and collagen accumulation, compared with transfection of an empty vector control. More important, STAT3-C expression abolished the inhibitory effects of HSP72 on fibroblast activation (Figure 6C), indicating that TGF- $\beta 1-$ mediated fibroblast activation occurs as a consequence of STAT3 phosphorylation, and modulation of these processes by HSP72 depends on the blockage of STAT3 phosphorylation.

\section{HSP72 Blocks Activation of the STAT3 Signal Pathway}

Next, we investigated mechanisms of the impact of HSP72 on the STAT3 signal pathway. The peak of p-STAT3 


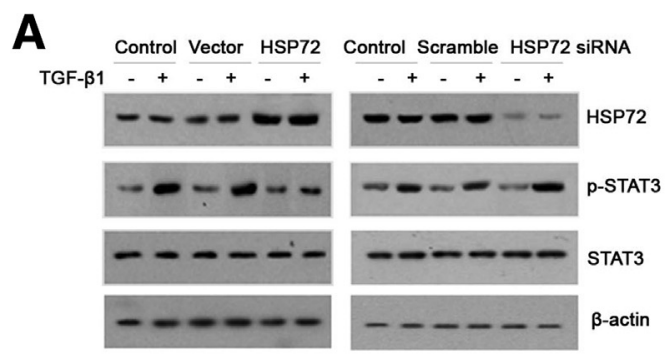

B

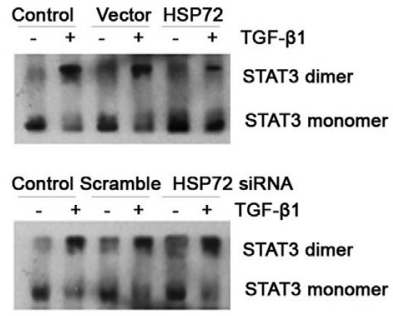

C
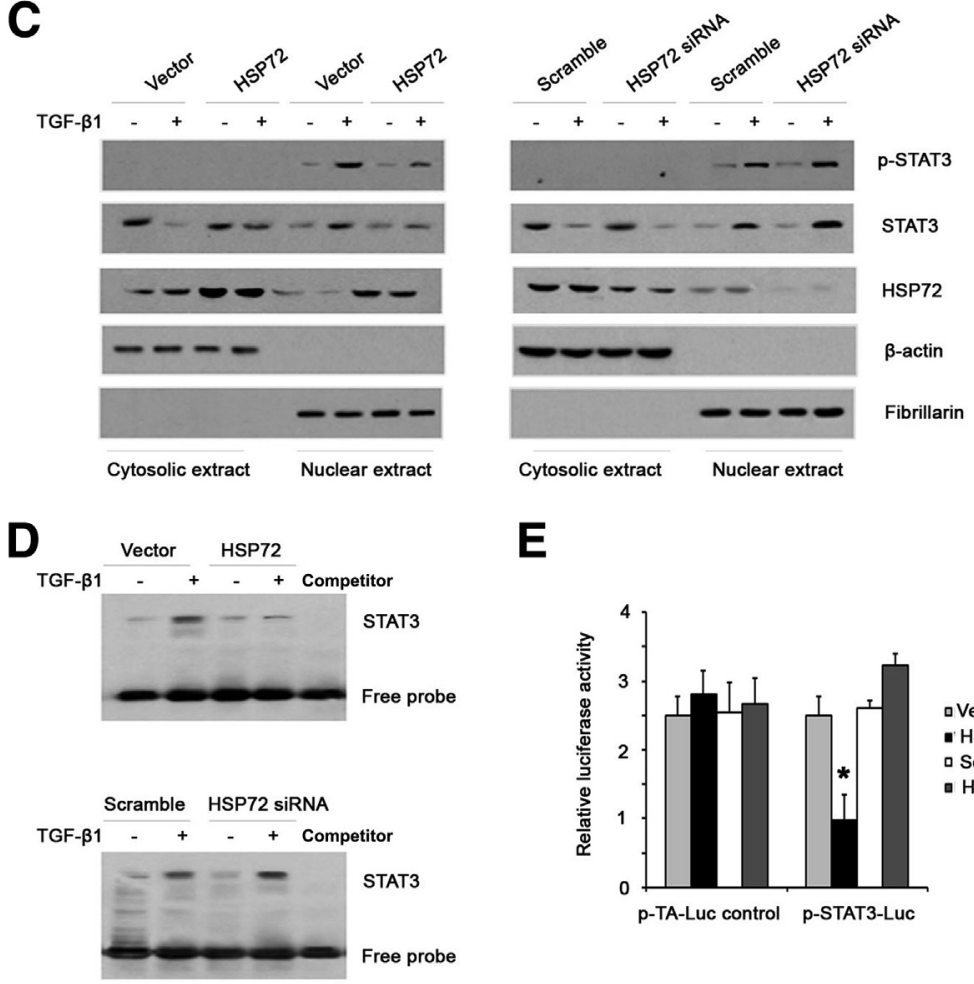

E

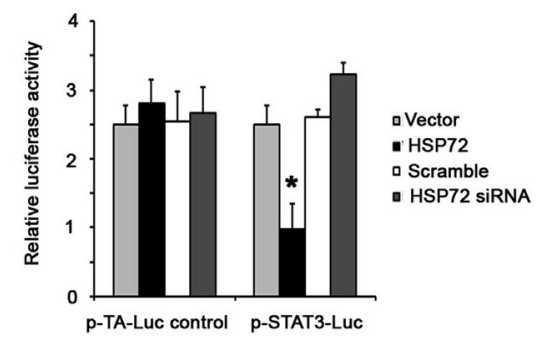

Figure 7 Heat shock protein (HSP) 72 suppresses STAT3 pathway. A: Cells were transfected with either wild-type (wt)-HSP72 or HSP72 siRNA, followed by $2 \mathrm{ng} / \mathrm{mL}$ of TGF- $\beta 1$ for 1 hour. Empty vector or scramble siRNA served as negative control. HSP72, p-STAT3, STAT3, and $\beta$-actin were evaluated by Western blot analysis. B: Native protein extracts were prepared from NRK- $49 \mathrm{~F}$ cells treated with various treatments. Proteins $(10 \mathrm{mg}$ per sample) were subjected to $6 \%$ native PAGE gel. Electrophoresis was performed in the absence of SDS. Proteins were immunoblotted with a STAT3 antibody. C: STAT3 and p-STAT3 nuclear translocations were assessed by immunoblot analysis. The expression of $\beta$-actin and fibrillarin served as the cytosolic and nuclear fraction controls, respectively. Results are representative of at least three separate experiments. D: Nuclear extracts were prepared from NRK-49F cells stimulated with $2 \mu \mathrm{g} / \mathrm{mL}$ of transforming growth factor (TGF)- $\beta 1$ for 1 hour in the presence of empty vector, wtHSP72, scramble siRNA, or HSP72 siRNA. The competition assays were conducted with nonbiotin-labeled STAT3-specific competitor at 100fold molar excess. The reaction mixtures were analyzed by electrophoretic mobility shift assay. E: Cells were transfected with either control p-TALuc plasmid or p-STAT3-Luc luciferase reporter plasmid under the control of STAT3 responsible element, and then cotransfected with empty vector, wt-HSP72, scramble, or HSP72 siRNA, followed by incubation with TGF- $\beta 1$ for 1 hour before luciferase activities were measured in cell extracts. ${ }^{*} P<0.05$ versus vector control, scramble, or HSP72 siRNA transfection. $n=3(\mathbf{E})$. appeared at 1 hour after incubation with TGF- $\beta 1$ (data not shown), which was chosen as the surrogate end point in subsequent experiments. Compared with empty vector and scrambled siRNA control, HSP72 overexpression led to a dramatic decrease in TGF- $\beta 1$-mediated STAT3 phosphorylation. Conversely, HSP72 siRNA resulted in a further significant increase of TGF- $\beta 1$-induced STAT3 phosphorylation (Figure 7A).

Phosphorylation of Tyr705 is known to promote dimerization of STAT3 and translocation to the nucleus, where it drives gene transcription. We thus assessed the STAT3 dimerization by using native PAGE. In agreement with the levels of p-STAT3, up-regulation of HSP72 decreased, but HSP72 siRNA enhanced, the amounts of dimerized STAT3 (Figure 7B) on TGF- $\beta 1$ exposure. As expected, overexpression of HSP72 prevented TGF- $\beta 1-$ induced STAT3 nuclear translocation, whereas HSP72 siRNA failed to block STAT3 nuclear accumulation compared with negative control (Figure 7C).

Because nuclear STAT3 acts as a transcriptional factor, binding to DNA at specific response elements and subsequently inducing gene expression, we examined whether HSP72 regulates the post-nuclear events of STAT3 signaling. The electrophoretic mobility shift assay showed that TGF- $\beta 1$ treatment increased STAT3 binding to DNA in NRK-49F cells. Consistently, the levels of STAT3 DNA binding activity were significantly reduced by up-regulation of HSP72, remained unchanged by down-regulation of HSP72 with siRNA, and were blocked by competitor oligonucleotide (Figure 7D). Furthermore, the inhibition of STAT3 transcriptional activity by HSP72 was confirmed by the reduced luciferase activity of p-STAT3-Luc by $60 \%$ in response to TGF- $\beta 1$ stimulation, compared with the p-TA-Luc control plasmid and HSP72 empty vector (Figure 7E). Taken together, our results indicate that HSP72 may be a negative regulator of the STAT3 signal pathway.

\section{Discussion}

Herein, we investigated the role of HSP72 in interstitial fibroblast activation, becoming myofibroblasts, and proliferation in 
the development of kidney fibrosis. We demonstrated an increase in fibroblast accumulation, HSP72 levels, and STAT3 phosphorylation in CKD patients with TIF. Moreover, we showed, for the first time to our knowledge, that enhanced HSP72 expression inhibited fibroblast proliferation and attenuated kidney fibrosis via targeting the STAT3 signaling pathway, whereas genetic deletion or pharmacological blockage of HSP72 eliminated those renoprotective effects.

Fibroblasts are interstitial mesenchyme that bridge the spaces between the tubules and capillaries and form a continuous network throughout the kidney. Renal fibrosis is an ultimate common pathological scarring process, triggered by non-specified chronic injury. Previous studies from various animal models and human kidney disease have suggested that activation of interstitial fibroblast to myofibroblast is the key step for ECM deposition in the kidney fibrosis. ${ }^{32,33}$ Indeed, we present evidence that the accumulation of $\alpha$-SMA-positive fibroblasts is exacerbated in the fibrotic kidney of animal models and the expression of $\alpha$ SMA in human samples correlates with the poor kidney function. Therefore, blocking fibroblast activation may finally halt progression of renal fibrogenesis regardless of etiology.

As a highly conserved family of molecular chaperones, HSP72 plays a protective role in a variety of disease models, including atherosclerosis, ${ }^{36}$ diabetes, ${ }^{37}$ ischemic renal injury, ${ }^{25}$ and renal fibrosis. ${ }^{26,27}$ It has also been shown that urinary HSP72 levels were increased in patients with stage 4 and 5 CKD compared with the healthy population. ${ }^{38}$ In the past decade, epithelial-mesenchymal transition has been studied extensively and indicated to promote kidney fibrosis development. Our previous studies have demonstrated that HSP72 prevents tubular cells from apoptosis and epithelialmesenchymal transition, and reduces renal fibrosis in rat kidneys after UUO injury. ${ }^{26,27}$ Nevertheless, the potential direct effect of HSP72 on renal fibroblast activation has never been addressed. HSP72 has been suggested to be a key molecule to participant in regulating proliferation and cell cycle progression. ${ }^{12,13,39}$ Both our in vitro and in vivo data show that overexpression of HSP72 inhibited renal fibroblast activation and proliferation, as evidenced by decreased fibrotic marker expression and a reduced number of fibroblasts, whereas genetic deletion or pharmacological blockage of HSP72 conversely eliminated these protective effects. Thus, our findings support the hypothesis that HSP72 is able to directly suppress renal fibroblast activation and proliferation.

A growing body of evidence has indicated that STAT3 activation is essential for proliferation and survival in tumor cell growth. ${ }^{40-42}$ It was also shown that inhibition of STAT3 signaling with a specific inhibitor attenuated fibroblast activation and renal fibrosis in obstructive nephropathy. ${ }^{20}$ Although the p38 MAPK pathway has been demonstrated in ECM synthesis in hepatic cells, dermal fibroblasts, and mesangial cells in vitro, ${ }^{43-45}$ the direct evidence of a role for p38 MAPK signaling in renal fibroblast activation and proliferation is lacking. Our in vitro results showed that STAT3, but not Akt or p38, activation was responsible for fibroblast activation and proliferation. Moreover, STAT3 activity correlated with fibroblast accumulation in both fibrotic kidneys of animal models and CKD patients, indicating that a STAT3-dependent mechanism is involved in this process.

Our study was the first time to demonstrate that HSP72 directly inhibited fibroblast activation and proliferation by targeting the STAT3 signaling pathway. Although endogenous HSP72 generation enhanced in diseased human kidneys, the increased HSP72 content might be not sufficient to counteract the effects of p-STAT3 on fibroblast activation and thereby renal fibrosis. Indeed, enhanced HSP72 expression appears to reduce STAT3 phosphorylation in vitro, whereas genetic deletion of HSP72 aggravated STAT3 activation in three animal models of kidney fibrosis. These findings indicate that forced overexpression of HSP72 may offer additional protection during stress. Blockage of STAT3 phosphorylation was essential for HSP72 to inhibit fibroblast activation, because constitutively expressing active form of STAT3 neutralized HSP72's protective role in suppression of $\alpha$-SMA expression and ECM accumulation in NRK-49F cells. In the present study, results also revealed that up-regulation of HSP72 decreased, but HSP72 siRNA increased, the amounts of dimerized STAT3 and nuclear translocation. In addition, STAT3 DNA binding activity declined and became almost undetectable in the overexpressed HSP72 fibroblast. Proliferation is regulated by cell cycle regulators. Cyclin D1 and p21 are critical mediators of $\mathrm{G}_{1}$ progression. Consistently, enhanced HSP72 protein expression was associated with changes in downstream targets of STAT3. HSP72 reduced cyclin D1 and increased p21 protein content, which paralleled decreased fibroblast number and bromodeoxyuridine incorporation. Because STAT3 phosphorylation is a prerequisite for its nuclear translocation and downstream signaling, it is conceivable that HSP72 inhibits TGF- $\beta 1$-induced p-STAT3 nuclear translocation, at least in part through interfering with its phosphorylation. However, the possibility of partial direct mechanical segregation of p-STAT3 or/and STAT3 preventing migration toward nucleus by HSP72 cannot be excluded. Taken together, our studies emphasize not only that the inhibiting effects of HSP72 on fibroblast activation and proliferation appear to rely on blocking STAT3 activation and its downstream signaling, but also that the STAT3 pathway may serve as a potential therapeutic target for kidney fibrosis.

Our previous studies and others have demonstrated that HSP72 protects renal tubular cells against various kinds of stress. $^{22,24-27}$ In this study, in addition to renal myofibroblasts, expression of HSP72 and p-STAT3 was also observed in tubular cells of the diseased human kidneys. Consistently, in the animal models of renal fibrosis, HSP72 enhancement by GGA reduced activation of STAT3 not only in the myofibroblasts, but also in the renal tubular cells, 
whereas HSP72 deficiency showed a significant increase in p-STAT3 in both cell types. A body of evidence showed that damage to renal tubular cells resulted in secretion of profibrogenic growth factors that caused fibroblast proliferation. $^{31,46,47}$ HSP72 has the capacity to modulate production of inflammatory cytokines in different cells and tissues and exert cellular protection. ${ }^{48-51}$ Thus, HSP72 appears to reduce the interplay between renal tubular cells and fibroblasts by attenuating a release of profibrotic factors from injured tubular cells, despite whether epithelial mesenchymal transition occurs. These findings indicate that HSP72 blockage of STAT3 activation can take place in different cell types and thereby curb renal fibrosis from the different levels.

In summary, we have demonstrated that HSP72, an endogenous molecule, directly targets fibroblast activation and proliferation to inhibit renal fibrosis via blocking the STAT3 signaling pathway. Future studies are needed to elucidate the possible therapeutic efficacy of HSP72 in slowing the progression of renal fibrosis.

\section{Supplemental Data}

Supplemental material for this article can be found at http://dx.doi.org/10.1016/j.ajpath.2015.11.016.

\section{References}

1. LeBleu VS, Taduri G, O'Connell J, Teng Y, Cooke VG, Woda C, Sugimoto H, Kalluri R: Origin and function of myofibroblasts in kidney fibrosis. Nat Med 2013, 19:1047-1053

2. Strutz F: How many different roads may a cell walk down in order to become a fibroblast? J Am Soc Nephrol 2008, 19:2246-2248

3. Humphreys BD, Lin SL, Kobayashi A, Hudson TE, Nowlin BT, Bonventre JV, Valerius MT, McMahon AP, Duffield JS: Fate tracing reveals the pericyte and not epithelial origin of myofibroblasts in kidney fibrosis. Am J Pathol 2010, 176:85-97

4. Ok CY, Chen J, Xu-Monette Z, Tzankov A, Manyam G, Li L, Visco C, Montes-Moreno S, Dybkaer K, Chiu A, Orazi A, Zu Y, Bhagat G, Richards KL, Hsi ED, Choi WW, van Krieken H, Huh J, Zhao X, Ponzoni M, Ferreri AJ, Bertoni F, Farnen JP, Moller MB, Piris MA, Winter JN, Medeiros LJ, Young KH: Clinical implications of phosphorylated STAT3 expression in de novo diffuse large B-cell lymphoma. Clin Cancer Res 2014, 20:5113-5123

5. Grivennikov S, Karin E, Terzic J, Mucida D, Yu GY, Vallabhapurapu S, Scheller J, Rose-John S, Cheroutre H, Eckmann L, Karin M: IL-6 and Stat3 are required for survival of intestinal epithelial cells and development of colitis-associated cancer. Cancer Cell 2009, 15:103-113

6. Zhang L, Zhou F, ten Dijke P: Signaling interplay between transforming growth factor-beta receptor and PI3K/AKT pathways in cancer. Trends Biochem Sci 2013, 38:612-620

7. Loesch M, Chen G: The p38 MAPK stress pathway as a tumor suppressor or more? Front Biosci 2008, 13:3581-3593

8. Koul HK, Pal M, Koul S: Role of p38 MAP kinase signal transduction in solid tumors. Genes Cancer 2013, 4:342-359

9. Judd LM, Bredin K, Kalantzis A, Jenkins BJ, Ernst M, Giraud AS: STAT3 activation regulates growth, inflammation, and vascularization in a mouse model of gastric tumorigenesis. Gastroenterology 2006, 131:1073-1085
10. Bromberg JF, Wrzeszczynska MH, Devgan G, Zhao Y, Pestell RG, Albanese C, Darnell JE Jr: Stat3 as an oncogene. Cell 1999, 98: 295-303

11. Darnell JE Jr: STATs and gene regulation. Science 1997, 277: $1630-1635$

12. Gong W, Wang L, Yao JC, Ajani JA, Wei D, Aldape KD, Xie K, Sawaya R, Huang S: Expression of activated signal transducer and activator of transcription 3 predicts expression of vascular endothelial growth factor in and angiogenic phenotype of human gastric cancer. Clin Cancer Res 2005, 11:1386-1393

13. Chalmin F, Ladoire S, Mignot G, Vincent J, Bruchard M, RemyMartin JP, Boireau W, Rouleau A, Simon B, Lanneau D, De Thonel A, Multhoff G, Hamman A, Martin F, Chauffert B, Solary E, Zitvogel L, Garrido C, Ryffel B, Borg C, Apetoh L, Rebe C, Ghiringhelli F: Membrane-associated Hsp72 from tumor-derived exosomes mediates STAT3-dependent immunosuppressive function of mouse and human myeloid-derived suppressor cells. J Clin Invest 2010, 120:457-471

14. Feng $\mathrm{X}$, Lu TC, Chuang PY, Fang W, Ratnam K, Xiong H, Ouyang X, Shen Y, Levy DE, Hyink D, Klotman M, D'Agati V, Iyengar R, Klotman PE, He JC: Reduction of Stat3 activity attenuates HIV-induced kidney injury. J Am Soc Nephrol 2009, 20:2138-2146

15. Benekli M, Xia Z, Donohue KA, Ford LA, Pixley LA, Baer MR, Baumann H, Wetzler M: Constitutive activity of signal transducer and activator of transcription 3 protein in acute myeloid leukemia blasts is associated with short disease-free survival. Blood 2002, 99:252-257

16. Lee JJ, Kim HJ, Yang CS, Kyeong HH, Choi JM, Hwang DE, Yuk JM, Park K, Kim YJ, Lee SG, Kim D, Jo EK, Cheong HK, Kim HS: A high-affinity protein binder that blocks the IL-6/STAT3 signaling pathway effectively suppresses non-small cell lung cancer. Mol Ther 2014, 22:1254-1265

17. Chong HC, Chan JS, Goh CQ, Gounko NV, Luo B, Wang X, Foo S, Wong MT, Choong C, Kersten S, Tan NS: Angiopoietin-like 4 stimulates STAT3-mediated iNOS expression and enhances angiogenesis to accelerate wound healing in diabetic mice. Mol Ther 2014, 22:1593-1604

18. Pang M, Ma L, Gong R, Tolbert E, Mao H, Ponnusamy M, Chin YE, Yan H, Dworkin LD, Zhuang S: A novel STAT3 inhibitor, S3I-201, attenuates renal interstitial fibroblast activation and interstitial fibrosis in obstructive nephropathy. Kidney Int 2010, 78:257-268

19. Powers MV, Clarke PA, Workman P: Dual targeting of HSC70 and HSP72 inhibits HSP90 function and induces tumor-specific apoptosis. Cancer Cell 2008, 14:250-262

20. Gupta S, Deepti A, Deegan S, Lisbona F, Hetz C, Samali A: HSP72 protects cells from ER stress-induced apoptosis via enhancement of IRE1alpha-XBP1 signaling through a physical interaction. PLoS Biol 2010, 8:e1000410

21. Reuter S, Bangen P, Edemir B, Hillebrand U, Pavenstadt H, Heidenreich S, Lang D: The HSP72 stress response of monocytes from patients on haemodialysis is impaired. Nephrol Dial Transplant 2009, 24:2838-2846

22. Mao H, Wang Y, Li Z, Ruchalski KL, Yu X, Schwartz JH, Borkan SC: Hsp72 interacts with paxillin and facilitates the reassembly of focal adhesions during recovery from ATP depletion. J Biol Chem 2004, 279:15472-15480

23. Marzec L, Liberek T, Chmielewski M, Bryl E, Witkowski JM, Liberek K, Zdrojewski Z, Rutkowski B: Expression of heat shock protein 72 in peritoneal leukocytes is induced by peritoneal dialysis. Perit Dial Int 2007, 27:288-295

24. Suzuki S, Maruyama S, Sato W, Morita Y, Sato F, Miki Y, Kato S, Katsuno M, Sobue G, Yuzawa Y, Matsuo S: Geranylgeranylacetone ameliorates ischemic acute renal failure via induction of Hsp70. Kidney Int 2005, 67:2210-2220

25. Wang Z, Gall JM, Bonegio RG, Havasi A, Hunt CR, Sherman MY, Schwartz JH, Borkan SC: Induction of heat shock protein 70 inhibits ischemic renal injury. Kidney Int 2011, 79:861-870 
26. Mao H, Li Z, Zhou Y, Li Z, Zhuang S, An X, Zhang B, Chen W, Nie J, Wang Z, Borkan SC, Wang Y, Yu X: HSP72 attenuates renal tubular cell apoptosis and interstitial fibrosis in obstructive nephropathy. Am J Physiol Renal Physiol 2008, 295:F202-F214

27. Zhou Y, Mao H, Li S, Cao S, Li Z, Zhuang S, Fan J, Dong X, Borkan SC, Wang Y, Yu X: HSP72 inhibits Smad3 activation and nuclear translocation in renal epithelial-to-mesenchymal transition. J Am Soc Nephrol 2010, 21:598-609

28. K/DOQI clinical practice guidelines for chronic kidney disease: evaluation, classification, and stratification. Am J Kidney Dis 2002, 39:S1-S266

29. Li S, Zhou Y, Fan J, Cao S, Cao T, Huang F, Zhuang S, Wang Y, Yu X, Mao H: Heat shock protein 72 enhances autophagy as a protective mechanism in lipopolysaccharide-induced peritonitis in rats. Am J Pathol 2011, 179:2822-2834

30. Park KM, Byun JY, Kramers C, Kim JI, Huang PL, Bonventre JV: Inducible nitric-oxide synthase is an important contributor to prolonged protective effects of ischemic preconditioning in the mouse kidney. J Biol Chem 2003, 278:27256-27266

31. Yang L, Besschetnova TY, Brooks CR, Shah JV, Bonventre JV: Epithelial cell cycle arrest in G2/M mediates kidney fibrosis after injury. Nat Med 2010, 16:535-543. 531p following 143

32. Kriz W, Kaissling B, Le Hir M: Epithelial-mesenchymal transition (EMT) in kidney fibrosis: fact or fantasy? J Clin Invest 2011, 121: 468-474

33. Kaissling B, Le Hir M: The renal cortical interstitium: morphological and functional aspects. Histochem Cell Biol 2008, 130:247-262

34. Ishii Y, Kwong JM, Caprioli J: Retinal ganglion cell protection with geranylgeranylacetone, a heat shock protein inducer, in a rat glaucoma model. Invest Ophthalmol Vis Sci 2003, 44:1982-1992

35. Strahl BD, Allis CD: The language of covalent histone modifications. Nature 2000, 403:41-45

36. Pockley AG: Heat shock proteins, inflammation, and cardiovascular disease. Circulation 2002, 105:1012-1017

37. Karpe PA, Tikoo K: Heat shock prevents insulin resistance-induced vascular complications by augmenting angiotensin-(1-7) signaling. Diabetes 2014, 63:1124-1139

38. Lebherz-Eichinger D, Ankersmit HJ, Hacker S, Hetz H, Kimberger O, Schmidt EM, Reiter T, Horl WH, Haas M, Krenn CG, Roth GA: HSP27 and HSP70 serum and urine levels in patients suffering from chronic kidney disease. Clin Chim Acta 2012, 413: 282-286

39. Tacke RS, Tosello-Trampont A, Nguyen V, Mullins DW, Hahn YS: Extracellular hepatitis C virus core protein activates STAT3 in human monocytes/macrophages/dendritic cells via an IL-6 autocrine pathway. J Biol Chem 2011, 286:10847-10855

40. Zhang Q, Yang Z, Jia Z, Liu C, Guo C, Lu H, Chen P, Ma K, Wang W, Zhou C: ISL-1 is overexpressed in non-Hodgkin lymphoma and promotes lymphoma cell proliferation by forming a p-STAT3/ p-c-Jun/ISL-1 complex. Mol Cancer 2014, 13:181

41. Qian Y, Chai S, Liang Z, Wang Y, Zhou Y, Xu X, Zhang C, Zhang M, Si J, Huang F, Huang Z, Hong W, Wang K: KIF5B-RET fusion kinase promotes cell growth by multilevel activation of STAT3 in lung cancer. Mol Cancer 2014, 13:176

42. Bollrath J, Phesse TJ, von Burstin VA, Putoczki T, Bennecke M, Bateman T, Nebelsiek T, Lundgren-May T, Canli O, Schwitalla S, Matthews V, Schmid RM, Kirchner T, Arkan MC, Ernst M, Greten FR: Gp130-mediated Stat3 activation in enterocytes regulates cell survival and cell-cycle progression during colitis-associated tumorigenesis. Cancer Cell 2009, 15:91-102

43. Varela-Rey M, Montiel-Duarte C, Oses-Prieto JA, LopezZabalza MJ, Jaffrezou JP, Rojkind M, Iraburu MJ: p38 MAPK mediates the regulation of alpha1(I) procollagen mRNA levels by TNF-alpha and TGF-beta in a cell line of rat hepatic stellate cells(1). FEBS Lett 2002, 528:133-138

44. Sato M, Shegogue D, Gore EA, Smith EA, McDermott PJ, Trojanowska M: Role of p38 MAPK in transforming growth factor beta stimulation of collagen production by scleroderma and healthy dermal fibroblasts. J Invest Dermatol 2002, 118:704-711

45. Chin BY, Mohsenin A, Li SX, Choi AM, Choi ME: Stimulation of pro-alpha(1)(I) collagen by TGF-beta(1) in mesangial cells: role of the p38 MAPK pathway. Am J Physiol Renal Physiol 2001, 280: F495-F504

46. Yang Y, Zhang ZX, Lian D, Haig A, Bhattacharjee RN, Jevnikar AM: IL-37 inhibits Il-18-induced tubular epithelial cell expression of pro-inflammatory cytokines and renal ischemiareperfusion injury. Kidney Int 2015, 87:396-408

47. Bonventre JV, Zuk A: Ischemic acute renal failure: an inflammatory disease? Kidney Int 2004, 66:480-485

48. Tang D, Kang R, Xiao W, Wang H, Calderwood SK, Xiao X: The anti-inflammatory effects of heat shock protein 72 involve inhibition of high-mobility-group box1 release and proinflammatory function in macrophages. J Immunol 2007, 179:1236-1244

49. Dokladny K, Lobb R, Wharton W, Ma TY, Moseley PL: LPSinduced cytokine levels are repressed by elevated expression of HSP70 in rats: possible role of NF-kappaB. Cell Stress Chaperones 2010, 15:153-163

50. Weiss YG, Bromberg Z, Raj N, Raphael J, Goloubinoff P, BenNeriah Y, Deutschman CS: Enhanced heat shock protein 70 expression alters proteasomal degradation of IkappaB kinase in experimental acute respiratory distress syndrome. Crit Care Med 2007, 35:2128-2138

51. Afrazi A, Sodhi CP, Good M, Jia H, Siggers R, Yazji I, Ma C, Neal MD, Prindle T, Grant ZS, Branca MF, Ozolek J, Chang EB, Hackam DJ: In tracellular heat shock protein-70 negatively regulates TLR4 signaling in the newborn intestinal epithelium. J Immunol 2012, 188:4543-4557 